\title{
Cost-effectiveness of dihydroartemisinin- piperaquine compared with artemether- lumefantrine for treating uncomplicated malaria in children at a district hospital in Tanzania
}

\author{
Amani T Mori ${ }^{1,2^{*}}$, Frida Ngalesoni ${ }^{1,3}$, Ole F Norheim ${ }^{1}$ and Bjarne Robberstad ${ }^{1}$
}

\begin{abstract}
Background: Dihydroartemisinin-piperaquine (DhP) is highly recommended for the treatment of uncomplicated malaria. This study aims to compare the costs, health benefits and cost-effectiveness of DhP and artemether-lumefantrine (AL) alongside "do-nothing" as a baseline comparator in order to consider the appropriateness of DhP as a first-line anti-malarial drug for children in Tanzania.

Methods: A cost-effectiveness analysis was performed using a Markov decision model, from a provider's perspective. The study used cost data from Tanzania and secondary effectiveness data from a review of articles from sub-Saharan Africa. Probabilistic sensitivity analysis was used to incorporate uncertainties in the model parameters. In addition, sensitivity analyses were used to test plausible variations of key parameters and the key assumptions were tested in scenario analyses.

Results: The model predicts that DhP is more cost-effective than AL, with an incremental cost-effectiveness ratio (ICER) of US\$ 12.40 per DALY averted. This result relies on the assumption that compliance to treatment with $\mathrm{DhP}$ is higher than that with AL due to its relatively simple once-a-day dosage regimen. When compliance was assumed to be identical for the two drugs, AL was more cost-effective than DhP with an ICER of US\$12.54 per DALY averted. DhP is, however, slightly more likely to be cost-effective compared to a willingness-to-pay threshold of US\$ 150 per DALY averted.

Conclusion: Dihydroartemisinin-piperaquine is a very cost-effective anti-malarial drug. The findings support its use as an alternative first-line drug for treatment of uncomplicated malaria in children in Tanzania and other sub-Saharan African countries with similar healthcare infrastructures and epidemiology of malaria.
\end{abstract}

Keywords: Tanzania, Dihydroartemisinin-piperaquine, Artemether-lumefantrine, Malaria, Cost-effectiveness, Markov model, Disability adjusted life years

\section{Background}

Malaria is an infectious disease which disproportionately affects pregnant women and children under the age of five years, and the disease is a major health problem in sub-Saharan Africa. In 2012, an estimated 627,000 deaths occurred due to malaria globally, mostly in

\footnotetext{
* Correspondence: pax_amani@yahoo.com

'Centre for International Health, Department of Global Public Health and Primary Care, University of Bergen, P.O. Box 7804, 5020 Bergen, Norway ${ }^{2}$ Muhimbili University of Health and Allied Sciences, P.O. Box 65001, Dar es Salaam, Tanzania

Full list of author information is available at the end of the article
}

African children under the age of five years [1]. Malaria accounts for $3.3 \%(82,685,000)$ of all Disability Adjusted Life Years (DALYs) and is ranked seventh among the top leading causes of DALYs globally [2]. Over the years, countries in sub-Saharan Africa have repeatedly changed their treatment policies in response to parasite resistance to monotherapy anti-malarials [3]. Recently, more expensive artemisinin-based combination therapy (ACT) has been recommended and have become increasingly common as first-line regimens against Plasmodium falciparum malaria $[1,3]$. 
The World Health Organization (WHO) recommends several artemisinin-based combinations for the treatment of uncomplicated malaria, including artesunate-sulphadoxine-pyrimethamine (ASSP), artesunateamodiaquine (ASAQ), artesunate-mefloquine (ASMQ), artemether-lumefantrine (AL) and dihydroartemisininpiperaquine (DhP) [4]. The newest ACT on this list is DhP, which has been proved to be more effective $[5,6]$, but is unfortunately also more expensive than AL, which is currently the most commonly used ACT in sub-Saharan Africa. Despite being more expensive, $\mathrm{DhP}$ has been recommended as a first-line or second-line alternative treatment for uncomplicated malaria [7-13].

In 2007, Tanzania changed its malaria treatment guidelines and adopted the use of AL as the first-line treatment for uncomplicated $P$. falciparum malaria to replace SP [14]. In 2013, the standard treatment guidelines were updated and DhP was officially adopted as the secondline drug for uncomplicated malaria [15]. AL has been shown to be a highly cost-effective first-line drug for the treatment of uncomplicated malaria $[16,17]$, but the cost-effectiveness evidence for DhP compared to AL is very limited [18].

Several countries in sub-Saharan Africa have officially adopted the use of DhP for the treatment of uncomplicated malaria $[19,20]$, and many others in the region are also contemplating this change. New drugs are typically more expensive than the existing alternatives: hence good trial results alone should not guarantee their inclusion in treatment guidelines as the additional health benefits may not be worth the extra costs. Pharmacoeconomic analyses are increasingly being used to generate evidence for decision-making in developing countries [21]. Therefore, this study aims to compare the costs, health benefits and cost-effectiveness of DhP and AL alongside "do-nothing" as a baseline comparator in order to consider the appropriateness of $\mathrm{DhP}$ as a first-line anti-malarial drug for children in Tanzania.

\section{Methods}

\section{Decision model}

Cost-effectiveness was analysed using a Markov decision model with four mutually exclusive health states: "well", "uncomplicated malaria", "severe malaria" and "death" (Figure 1). Newborn children are assumed to be protected from malaria through breastfeeding, and enter the model when they are six months old in a "well" state. In the model, they are tracked until they are five years old, after which they are assumed to have gained sufficient clinical immunity against malaria [22,23]. During this time, children move between the health states in oneweek cycles depending on risk factors, access to and effectiveness of anti-malarial treatments.

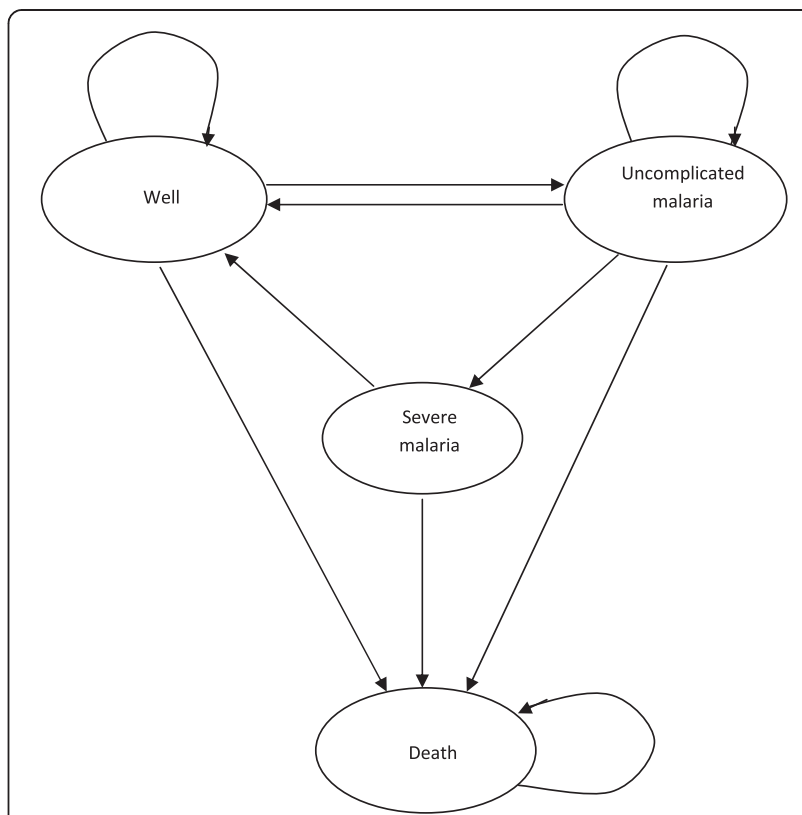

Figure 1 State-transition diagram of the model.

The model assumes that children first develop uncomplicated malaria, from which they may recover and return to the "well" state, or they may progress to "severe malaria", which requires hospitalization. "Death" is an absorbing health state, which may occur spontaneously (i.e. background mortality) or as an outcome of severe malaria. In each cycle the model captures and accumulates costs and utilities related to the patient's health state. Probabilistic Sensitivity Analyses (PSA) were based on a Monte Carlo simulation with 10,000 iterations using TreeAge Pro ${ }^{\odot} 2014$ software.

\section{Collection of cost data}

Cost data from a provider's perspective was collected at Mwananyamala Hospital in Dar- es Salaam region, from August to November 2012. This is an urban, districtlevel public hospital with about 400 beds and 400,000 visits per year. Costs were collected for the treatment of both uncomplicated and severe malaria in order to capture the additional costs for patients who develop severe malaria after unsuccessful treatment with the first-line drugs. A district hospital was chosen because it is the lowest level at which severe malaria can be managed effectively within the Tanzanian healthcare system. Costs represent the expenditures incurred during the financial year that ended on June $30^{\text {th }} 2012$ and were collected using an ingredient approach [24]. Costs were collected in the local currency and converted to US dollars (US\$ $1=1,578$ Tanzanian shillings) [25].

Four service centres were identified; namely the general outpatient department, general paediatric ward, pharmacy and the laboratory. Support departments, which included 
general administration and transportation, were categorized as overheads. Costs for resources which last longer than a year were categorized as capital costs and included furniture, equipment and motor vehicles. Recurrent costs were those incurred on resources that are purchased regularly and used up in the course of a year, and include salaries rental charges, utilities and supplies [26].

Cost data were recorded in a pre-tested questionnaire which was designed to capture all the necessary data, including the types and quantities of items, their sources, prices and allocation base. Functioning capital items were identified, counted and valued using their assumed replacement market prices. The price catalogue from the Medical Stores Department (MSD) was used to value medical items and supplies [27]. Capital costs were annuitized at a discount rate of $12 \%$ as recommended by the Bank of Tanzania [25] and their useful life years were adopted from the WHO-CHOICE Project [28].

Staff members were identified and interviewed in order to discover their monthly earnings, including gross salary and other standard remunerations. Salary scales and remunerations were cross-checked and validated by the hospital secretary. Personnel costs attributable to malaria were calculated by multiplying total staff monthly earnings by the percentage of their time devoted to malaria. For the buildings, floor spaces were measured and valued as per the square metre rental charges recommended by the National Housing Corporation.

The Global Fund's maximum manufacturer prices for ACTs that are financed through the Affordable Medicines Facility-malaria (AMFm) was used to estimate the mean cost of a course of treatment with AL and DhP [29]. For AL the " $6 \times 2$ " tablet pack specified for children weighing 15-24 kg was used [13] and the " $3 \times 1$ " tablet pack for children weighing 13-24 kg was used for DhP [30]. These prices were inflated by $10 \%$ to account for freight and insurance costs [31] and further by a domestic margin factor of 1.43 to represent local opportunity costs [32]. Prices of all the other drugs used in the management of malaria were taken from the MSD's Price Catalogue.

Each service department was allocated a portion of the overhead costs proportional to its percentage contribution to the total allocation base by using the direct-allocation method [24]. For example, cleaning costs were allocated based on floor space. Allocation was difficult for some expenditure, such as electricity, medical supplies, stationery, which were paid for centrally but for which usage was not specified by the departments. Therefore, some of the overhead costs were equally distributed between the departments while others were allocated using an estimated weightedallocation factor based on interviews with hospital management. For more details about personnel costs and rental charges, see Additional file 1.

The hospital has Health Management and Information System (HMIS) tools to keep records of all the attendances and diagnoses made during each year. However, because of poor recording, the attendances of malaria patients in the pharmacy or the laboratory could not be tracked. Therefore, the unit costs for the treatment of uncomplicated and severe malaria were calculated by dividing the total costs attributable to malaria for the service centres by the respective number of outpatients (7,076 cases) and hospitalized patients (1,263 cases) recorded in the HMIS tools during the year.

\section{Choice of health outcomes}

Disability Adjusted Life Years (DALYs), which combines years of life lost due to premature death (YLL) and years of life lived with disabilities (YLD), was used as a measure of health outcomes [2]. Disability weights of 0.005 and 0.21 for mild and severe acute episodes of infectious diseases from the recent Global Burden of Disease study were applied for uncomplicated and severe malaria, respectively [33]. DALYs averted were calculated using standard methods [34] as a difference of DALYs lost with and without the intervention, based on a life expectancy of 57 years at age 5 for Tanzania [35]. Base case DALYs were discounted at 3\%, without age-weighting. Results for age-weighted and undiscounted DALYs were reported in the scenario analysis.

\section{Interventions compared}

The study compares DhP (the potential new standard of care) and AL (the existing standard of care) alongside "do nothing" as a baseline comparator. Both drugs are administered for three consecutive days, but AL should be given twice a day with high-fat meals [36] while DhP is given once a day without the requirement for fatty meals [37]. Because of its relatively simple dosage regimen, it is likely that compliance with and hence the effectiveness of DhP will be higher than that of AL in clinical settings. DhP also offers a longer patient protection from re-infection with malaria because piperaquine has a significantly longer elimination halflife of 3-4 weeks compared to the 4-6 days of lumefantrine [38]. The impact of high compliance with DhP is included in the base-case scenario of our model, while that of longer protection is not.

\section{Measurement of effectiveness}

Patient compliance to treatment in routine clinical practice plays a key role in the effectiveness of anti-malarial therapies. Thus the effectiveness of each drug, $E_{f f}$, was 
calculated by combining efficacy and compliance rates using the equation below:

$$
\mathrm{E}_{\mathrm{ff}}=\mathrm{E}_{\mathrm{o}} \mathrm{C}+\mathrm{E}_{\mathrm{nc}}(1-\mathrm{C})
$$

Where $E_{o}$ is the efficacy, $C$ is the compliance rate and $E_{n c}$ is the proportion of non-compliers for whom treatment is effective, assumed to be $10-30 \%$, which has been employed in several other cost-effectiveness studies for ACT [39-41]. Efficacy data were extracted from a large, head-to-head, randomized clinical trial which was conducted among 6-59-month-old children in seven African countries with different malaria endemicities: Uganda, Zambia, Mozambique, Rwanda, Nigeria, Gabon and Burkina Faso. The study used the 28-day PCRcorrected cure rate of $97.3 \%$ for $\mathrm{DhP}$ and $95.5 \%$ for $\mathrm{AL}$, from the intention-to-treat analysis [13].

Evidence on compliance to ACT is very limited and diverse [42,43]; however, it has been reported that compliance to AL by "verified timely completion" ranges from 38 to $65 \%$ [43]. DhP is a new drug and evidence on its compliance is currently lacking. Since the potential benefit of its once-a-day dosage regimen consisting of only a few tablets is an improved compliance, a range of 60 to $80 \%$ was assumed in the base case analysis. This is a conservative assumption, considering that a compliance of $67-87 \%$ and $87.2-92.5 \%$ have been reported for co-blistered and fixed-dose ASAQ, among children in Tanzania and Madagascar, respectively [44,45]. ASAQ has a once-a-day dosing schedule similar to that of DhP. An assumed compliance similar to that of AL was explored in a scenario analysis.

\section{Transition probabilities}

Children enter the model in a "well" state, and can develop febrile episodes based on the estimated agespecific incidence rates shown in Table 1. All febrile children were assumed to be taken to the hospital for diagnosis, and $10.5 \%$ of the episodes were attributed to malaria [46]. Between 40 and $60 \%$ of children with uncomplicated malaria were assumed to have access to first-line drugs and the probability of cure depends on efficacy and compliance with treatment. Efficacies of AL and DhP were $95.5 \%$ and $97.3 \%$, [13] and the base line compliance rates ranged between $38-65 \%$ for AL [43] and $60-80 \%$ for DhP. The remaining children were assumed to be treated with over-the-counter non-ACT anti-malarials, with effectiveness ranging from 40 to $60 \%[47,48]$.

In the "do-nothing" arm, between 3-7\% of uncomplicated malaria cases progress to severe malaria [40], which has been estimated to have a case fatality rate ranging from 45 to $80 \%$ [51]. Between 10 to $20 \%$ of the uncomplicated malaria cases were assumed to recover spontaneously without treatment. In the DhP and AL arms, about $3-7 \%$ of the uncomplicated malaria cases progress to severe malaria in the event of treatment failure [41], of whom between $72-88 \%$ were assumed to have prompt access to inpatient care [53], which reduces case-fatality rate to $10.9 \%$ [52]. Besides malaria, children can also die of other causes at any state in the model based on adjusted age-specific probabilities of death taken from the Tanzanian Life Table [35].

\section{Sensitivity and specificity of the test}

Bayesian method was used to incorporate the sensitivity and specificity parameters of the microscopic test in the model, which have been estimated to be $71.3 \%$ (95\% CI: 68.8-73.9) and 92.8\% (95\% CI: 91.3-94.3), respectively [56]. Rate of adherence by clinicians to negative test results was estimated to range from 40 to $60 \%[56]$.

\section{Model simplifications}

The model is a simplification of a complex disease with complex treatment-seeking behaviour and management practices. It is based on the following simplifying assumptions:

- A child cannot move directly from a "well" to "severe malaria" state, but severe malaria is always a progression from uncomplicated malaria.

- Uncomplicated malaria is not fatal, hence a child cannot move from "uncomplicated malaria" to the "death" state, except for deaths caused by other reasons (i.e. background mortality).

- In the event of treatment failure, patients with uncomplicated malaria will repeatedly use the same first-line drug, which we assumed will still be effective.

\section{Uncertainty and sensitivity analyses}

Uncertainties in parameters were included in the model by using probability distributions (Table 1). Maximum and minimum values for each parameter were taken from the literature and when these were not available, the mean values were varied by $+/-20 \%$ and efficacy data by $+/-2.5 \%$. The gamma distribution was used to constrain costs on the $[0,+\infty]$ interval and the beta distribution to fix the probabilities on the $[0,1]$ interval. Gamma and beta distributions were calculated using the method of moments [57]. Uncertainty in the PSA results is presented using a cost-effectiveness acceptability curve (CEAC). Sensitivity and scenario analyses were also performed to assess the influence of variations in the key parameters. 
Table 1 Parameters used in the economic model and their distributions

\begin{tabular}{|c|c|c|c|}
\hline Parameters & Estimates & Distributions & Sources \\
\hline \multicolumn{4}{|l|}{ Age-specific probabilities of death } \\
\hline Probability of dying between 0 and 1 year & $0.0684 \pm 20 \%$ & Beta & {$[35]$} \\
\hline Probability of dying between 1 and 5 years & $0.0424 \pm 20 \%$ & Beta & {$[35]$} \\
\hline Malaria-attributed deaths in under fives & $11 \%$ & Point estimate & [49] \\
\hline \multicolumn{4}{|l|}{ Weekly incidences of fever episodes per child } \\
\hline Less than 12 months & $0.106 \pm 20 \%$ & Beta & {$[50]$} \\
\hline Age $12-23$ months & $0.144 \pm 20 \%$ & Beta & {$[50]$} \\
\hline Age $24-35$ months & $0.105 \pm 20 \%$ & Beta & {$[50]$} \\
\hline Age $36-47$ months & $0.087 \pm 20 \%$ & Beta & {$[50]$} \\
\hline Age $48-59$ months & $0.06 \pm 20 \%$ & Beta & {$[50]$} \\
\hline \multicolumn{4}{|l|}{ Case fatality rates and other probabilities } \\
\hline Untreated severe malaria & $60(45-80 \%)$ & Beta & {$[51]$} \\
\hline Treated severe malaria & $10.9 \%$ & Beta & {$[52]$} \\
\hline Early treatment failure leads to severe malaria & $5(3-7 \%)$ & Beta & {$[41]$} \\
\hline Untreated malaria becomes severe & $5(3-7 \%)$ & Beta & {$[40]$} \\
\hline Spontaneous recovery from uncomplicated malaria & $15(10-20 \%)$ & Beta & Assumed \\
\hline$\%$ of febrile episodes attributed to malaria & $10.5 \pm 20 \%$ & Beta & {$[46]$} \\
\hline$\%$ of severe cases with access to inpatient care & $80 \pm 20 \%$ & Beta & {$[53]$} \\
\hline$\%$ of uncomplicated cases with access to AL & $50(40-60 \%)$ & Beta & Primary data \\
\hline \multicolumn{4}{|l|}{ Costs of treating malaria, by severity (US\$/case) } \\
\hline Uncomplicated malaria & $6.81 \pm 20 \%$ & Gamma & Primary data \\
\hline Severe malaria & $76.46 \pm 20 \%$ & Gamma & Primary data \\
\hline \multicolumn{4}{|l|}{ Drug costs (US\$ per dose) } \\
\hline DhP: 40 mg Dh, 320 mg P ("3×1" pack) & $1.46 \pm 20 \%$ & Gamma & {$[29]$} \\
\hline AL: 20 mg A, 120 mg L (“6×2" pack) & $1.31 \pm 20 \%$ & Gamma & [29] \\
\hline Quinine Injection, 300 mg/ml (2 ml ampoule) & $2.15 \pm 20 \%$ & Gamma & {$[27]$} \\
\hline Diazepam Injection, 5 mg/ml (2 ml ampoule) & $0.23 \pm 20 \%$ & Gamma & {$[27]$} \\
\hline Diclofenac Injection 25 mg/ml (3 ml ampoule) & $0.20 \pm 20 \%$ & Gamma & {$[27]$} \\
\hline Dextrose 5\% (500 ml bottle) & $4.75 \pm 20 \%$ & Gamma & {$[27]$} \\
\hline Ferrous Sulphate + Folic acid, $200+0.25$ mg & $0.30 \pm 20 \%$ & Gamma & {$[27]$} \\
\hline Paracetamol Syrup 120 mg/5 ml & $0.26 \pm 20 \%$ & Gamma & {$[27]$} \\
\hline \multicolumn{4}{|l|}{ Efficacy and compliance rates (\%) } \\
\hline Efficacy of DhP & $97.3 \pm 2.5 \%$ & Beta & [13] \\
\hline Efficacy of AL & $95.5 \pm 2.5 \%$ & Beta & {$[13]$} \\
\hline Effectiveness of non-ACT anti-malarials & $50(40-60 \%)$ & Beta & {$[47,48]$} \\
\hline Compliance with AL & $51(38-65 \%)$ & Uniform & [43] \\
\hline Compliance with $\mathrm{DhP}^{\mathrm{a}}$ & $70(60-80 \%)$ & Uniform & Assumed \\
\hline Compliance with DhPb & $51(38-65 \%)$ & Uniform & Assumed \\
\hline Non-compliers with ACTs who are cured & $20(10-30 \%)$ & Beta & {$[39-41]$} \\
\hline \multicolumn{4}{|l|}{ Other parameters } \\
\hline Disability weight for uncomplicated malaria & $0.005(0.033-0.081)$ & Beta & {$[33]$} \\
\hline Disability weight for severe malaria & $0.21(0.139-0.298)$ & Beta & [33] \\
\hline Discount rate & $3 \%$ & Point estimate & {$[54]$} \\
\hline Decision threshold (US\$ per DALY averted) & 150 & Point estimate & {$[55]$} \\
\hline
\end{tabular}


Table 1 Parameters used in the economic model and their distributions (Continued)

\begin{tabular}{llll}
\hline Life expectancy at age 5 years & 57 & Point estimate & [35] \\
Sensitivity of Microscopy & $71.3(68.8-73.9 \%)$ & Beta & [56] \\
Specificity of Microscopy & $92.8(91.3-94.3 \%)$ & Beta & [56] \\
\hline
\end{tabular}

${ }^{a}$ Used in the base case analysis, ${ }^{b}$ Used in the scenario analysis.

\section{Cost-effectiveness threshold}

An intervention that produces more health benefits at a lower cost than the comparator is considered to be "strongly dominant" and cost-effective. If it is more costly but also more effective, it is considered costeffective only when its incremental cost-effectiveness ratio (ICER) is less than the willingness-to-pay threshold. "Extended dominance" occurs when the ICER of an intervention is higher than that of the next most effective option [58]. A willingness-to-pay threshold of US\$ 150 per DALY averted, which has been recommended as a cut-off point for low- and middle-income countries was applied [55].

\section{Ethics statement}

This study was approved by the Ethical Review Committee of the Tanzania National Institute of Medical Research with clearance certificate no: NIMR/HQ/R.8a/Vol.IX/1362. The District Medical Officer in charge of Kinondoni and the management at Mwananyamala Hospital also gave permission to conduct the costing study. The interviewed health workers each provided written informed consent to participate in the study.

\section{Results}

Unit costs of treatment

Table 2 presents the estimated unit costs of treating cases of uncomplicated and severe malaria with the associated co-morbidities at an urban district-level hospital in Tanzania. For uncomplicated malaria, the cost per episode was US\$ 8.40 with AL and US\$ 8.54 with DhP. For severe malaria, the hospitalization cost per episode was estimated to be US\$ 83.86 .

Table 2 Unit costs (US\$) for outpatient and inpatient care

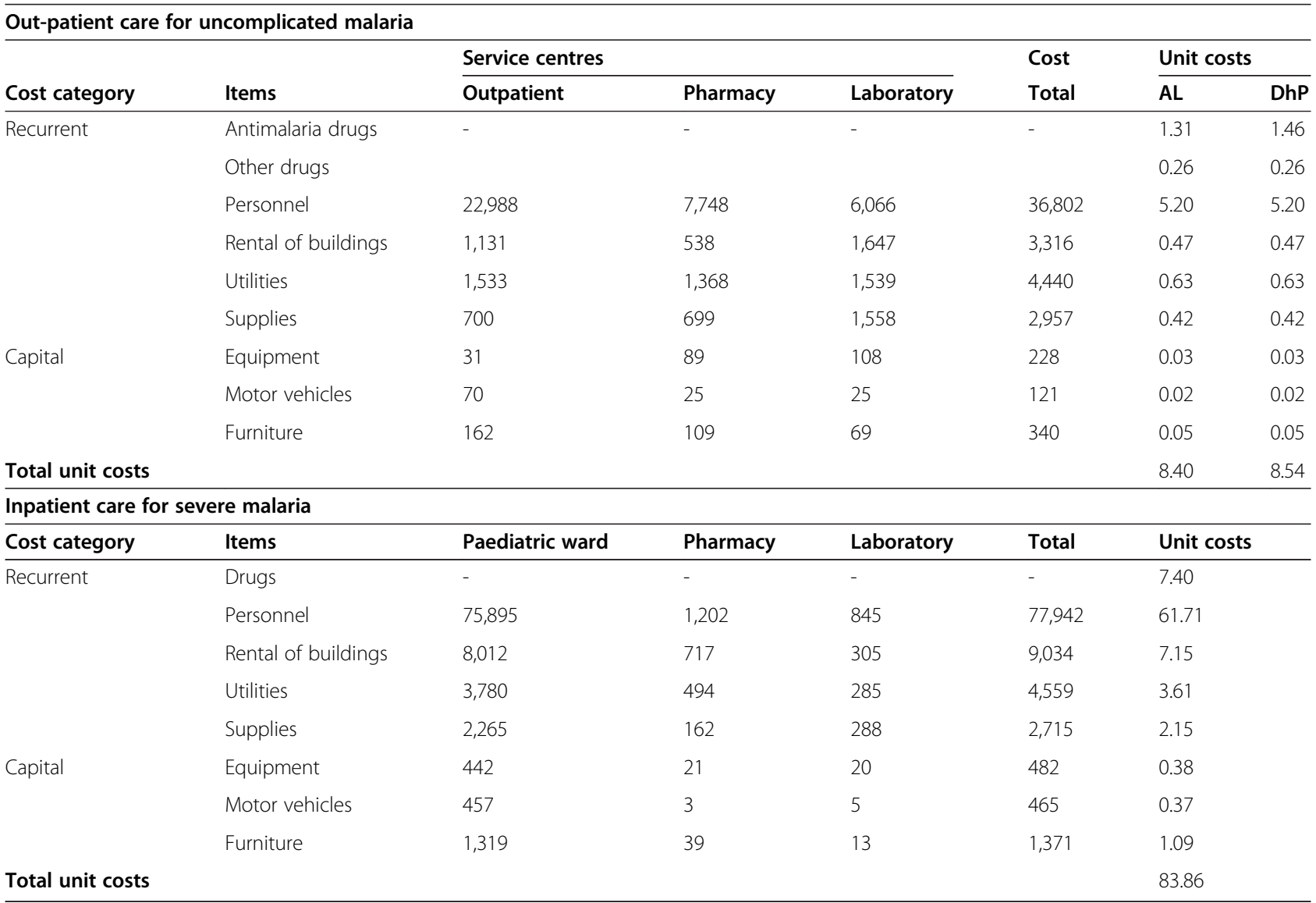




\section{Cost-effectiveness analysis}

Table 3 presents the base-case analysis, for which the model predicts that DhP is more cost-effective than AL, with an ICER of US\$ 12.40 per DALY averted. AL was eliminated in the base-case analysis because it was extendedly dominated by DhP, therefore, the base-case ICER value represents the comparison of DhP to a do nothing strategy. In the scenario assuming a lower compliance, similar to that of AL, ranging from 38-65\%, AL was more cost-effective than DhP with an ICER of US\$ 12.54 per DALY averted versus US\$ 101.52 per DALY averted.

\section{Incremental cost-effectiveness scatter plot}

Figure 2 shows the base-case ICE scatter plot of DhP versus AL. The model predicts that DhP is cost-effective in $97 \%$ of the simulations and dominated by AL in $2 \%$ of the simulations, at a willingness-to-pay threshold of US\$ 150 per DALY averted. With a compliance of 38-65\%, DhP was cost-effective in $51 \%$ of the simulations and dominated by AL in $37 \%$ of the simulations.

\section{Cost-effectiveness acceptability curve}

Figure 3 shows the cost-effectiveness acceptability curves (CEAC) for the base-case and scenario analyses of DhP compared to AL. For the base-case, the probability of DhP being cost-effective was $97 \%$ at the willingness-topay threshold of US\$ 150 per DALY averted. In the scenario analysis where we assumed the compliance with DhP to be $38-65 \%$, the probability of DhP being costeffective was $51 \%$ compared to $49 \%$ for AL at the same willingness-to-pay threshold of US\$ 150 per DALY averted

\section{Characterizing uncertainty}

One-way sensitivity analyses were conducted to assess the influence of plausible variations of key parameters on cost-effectiveness of DhP versus AL. The result shows that the cost-effectiveness of DhP relies on the assumption that it has a higher compliance rate than $\mathrm{AL}$, for which the evidence is weak. This is illustrated in Figure 4, which shows that when the compliance with DhP is assumed to be less than 50\% it produces fewer health benefits at higher costs than AL (strongly dominated) and at between 50 and 56\% it is less cost-effective than AL. When compliance exceeds a threshold of $57 \%$, DhP becomes the cost-effective strategy by extended dominance. Above 85\%, DhP produces more health benefits at a lower cost than AL (strong dominance). Note that the compliance rate for $\mathrm{AL}$ was held constant at $51 \%$.

Figure 5 shows a tornado diagram which ranks the parameters in the order of their decreasing influence on the base-line ICER value. In the diagram, DhP was compared to "do-nothing" because AL was eliminated in the analysis due to extended dominance. Uncertainties in parameters describing the natural history of malaria were the most influential on the ICER value. This includes the probability of progression to severe malaria (Untreated to SM), case fatality rate for severe malaria (CFR untreated SM) and the probability of "self-limiting" uncomplicated malaria. The cost-effectiveness of DhP increases with an increase in the values of the first two parameters but decreases with an increase in the probability of self-limiting malaria. The cost-effectiveness of DhP also increases with an increase in the incidence rates of malaria, making it a good choice in hightransmission areas.

\section{Two-way sensitivity analysis}

The existing evidence for compliance with AL is very diverse. We therefore performed a two-way sensitivity analysis (Figure 6), to determine various combinations of compliance rates at which the two drugs were costeffective, at a willingness-to-pay threshold of US\$ 150 per DALY averted. This shows that even when compliance is perfect for both drugs, DhP remains slightly more cost-effective than AL.

\section{Impact of age-weighting and discounting}

In the base-case analysis, DALYs were calculated without age-weighting and with a discount rate of $3 \%$. When DALYs were not discounted, the ICER value of DhP compared to "do-nothing" in the deterministic analysis decreased from US\$ 12.33 to 10.80 per DALY averted. Age-weighting assigns different values to time lived at different ages and when it was applied the ICER increased from US\$ 12.33 to 18.00 per DALY averted. None of these choices of method had any influence on the conclusions.

\section{Discussion}

This study has shown that DhP is a cost-effective antimalarial drug with an incremental cost-effectiveness

Table 3 Base-case cost-effectiveness analysis

\begin{tabular}{|c|c|c|c|c|c|}
\hline Strategy & Cost (US\$) & DALYs & Incremental cost & Incremental DALYs averted & ICER \\
\hline No treatment & 0.00 & 17.60 & 0.00 & 0.00 & 0.00 \\
\hline$A L$ & 165.42 & 4.47 & 165.42 & 13.13 & Extendedly dominated \\
\hline DhP & 166.22 & 4.22 & 0.80 & 0.25 & 12.40 \\
\hline
\end{tabular}




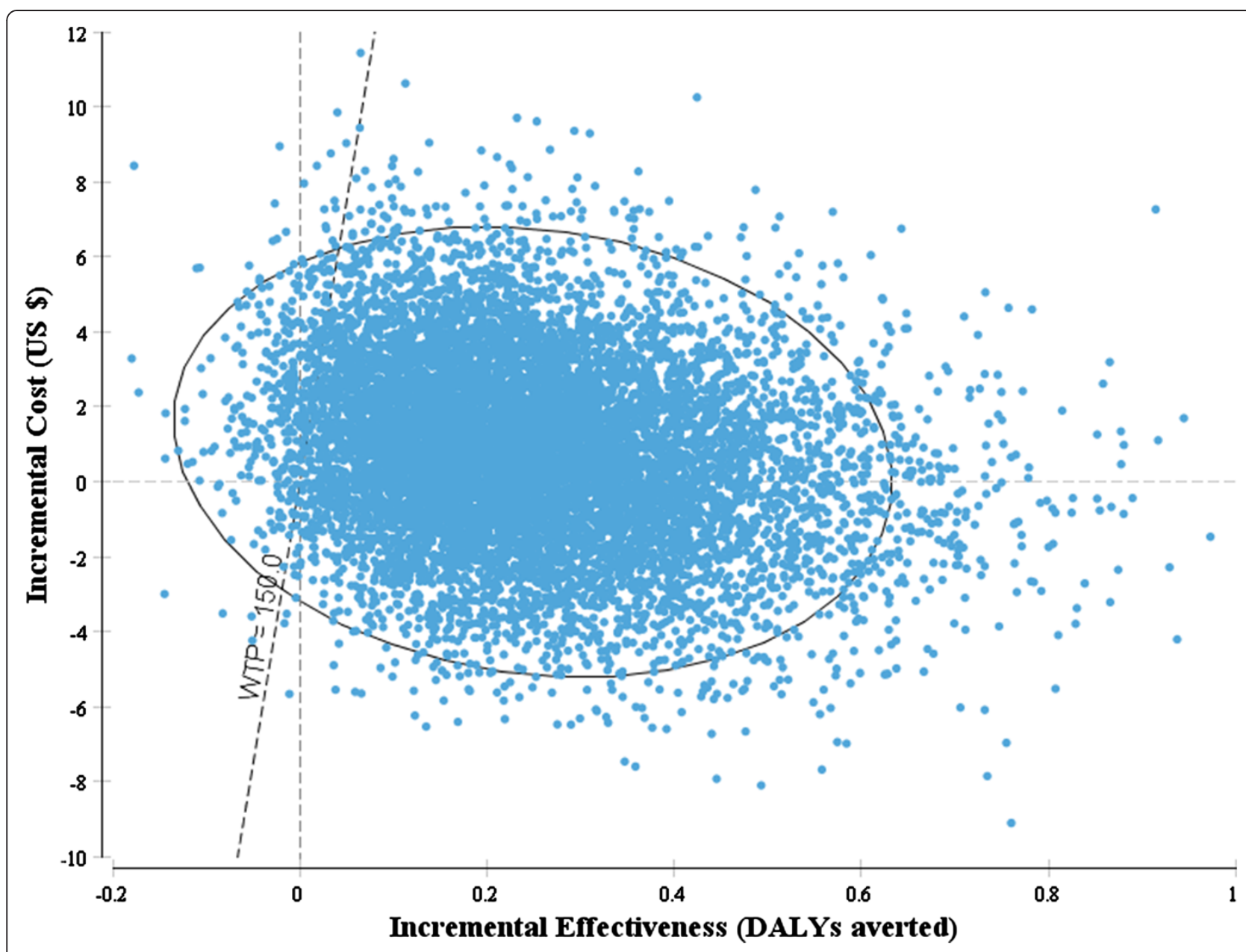

Figure 2 Incremental cost-effectiveness scatter plot DhP versus AL. Key: The dots represent incremental cost-effect pairs for DhP versus AL for 10,000 Monte Carlo simulations. The dotted line represents a willingness-to-pay threshold of US\$150 per DALY averted.

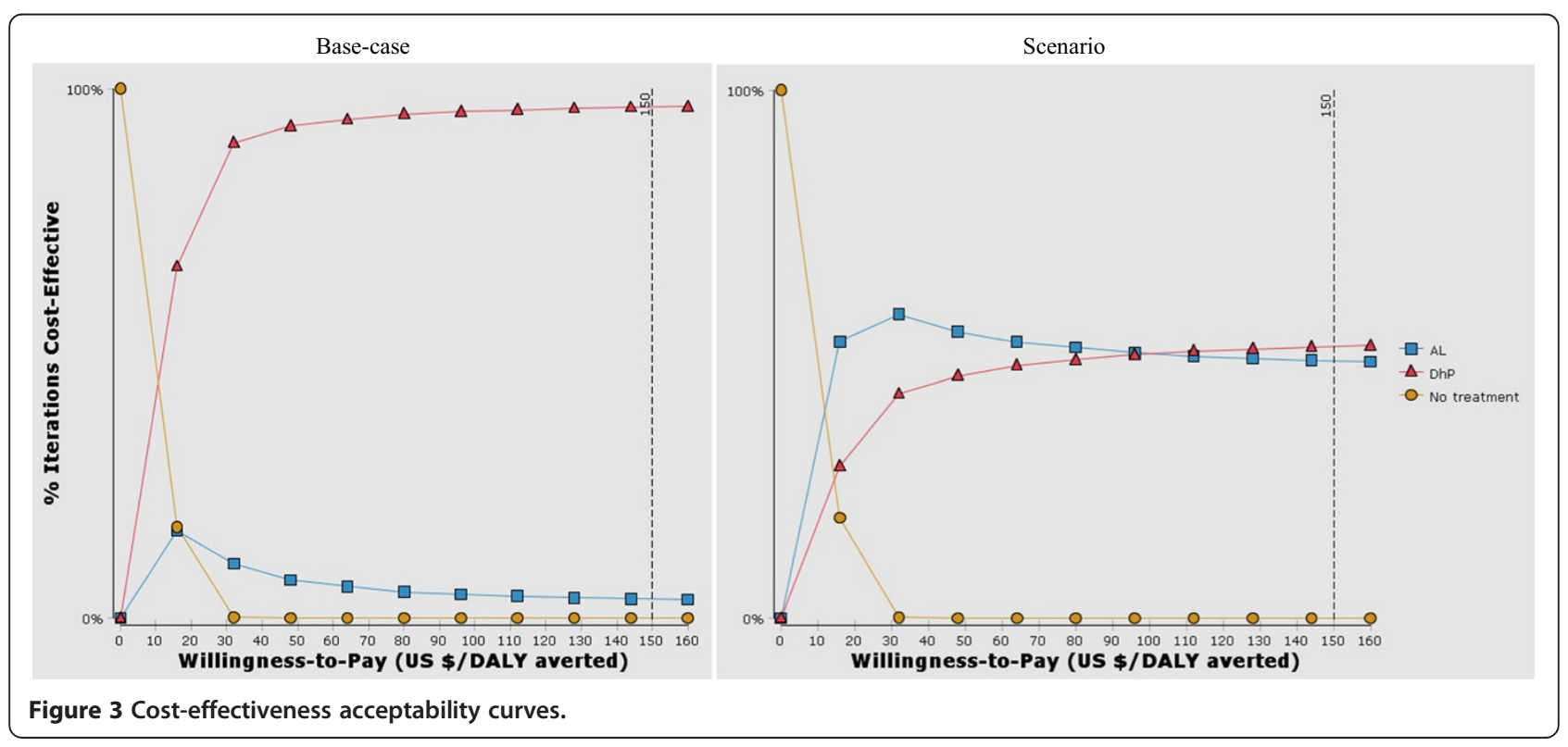




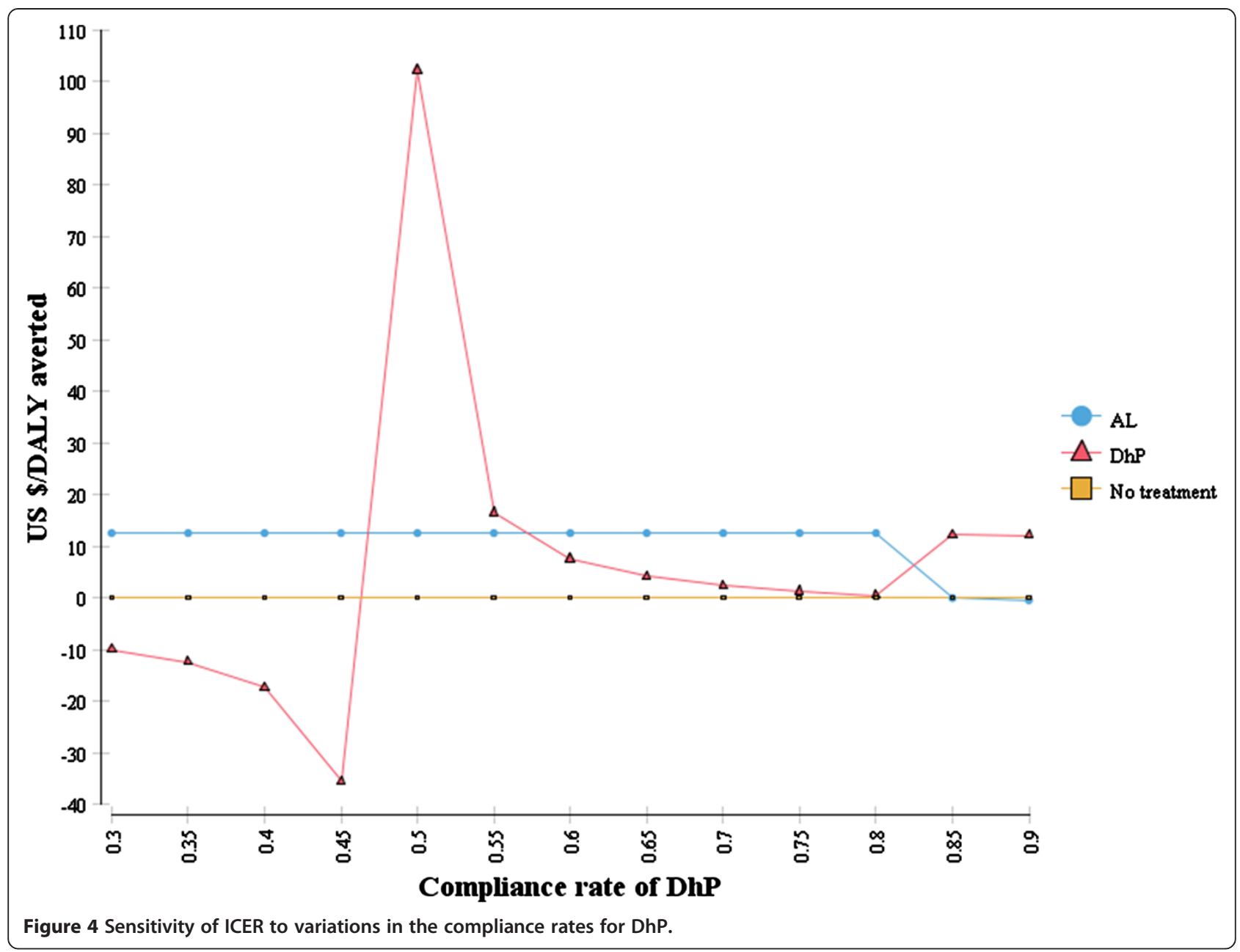

ratio of US\$ 12.40 per DALY averted compared to AL. This finding is higher than the US\$ 6.23 per DALY averted that was predicted by the Committee on the Economics of Anti-malarial Drugs, which compared ACT with "do-nothing", from the provider's perspective [40]. The ICER is well below all common rules of thumb for cost-effectiveness, including the GDP per capita for each DALY averted recommended by the WHO [59] and the US\$ 150 per DALY averted suggested for low- and middle-income countries [55]. Therefore, adequate and timely provision of DhP can be considered a highly costeffective treatment for uncomplicated malaria.

$\mathrm{DhP}$ is currently more expensive than AL and hence any decision to adopt it nationwide as a first-line drug will have significant budget implications. However, DhP has two major advantages over AL that make it an attractive weapon in the fight against malaria. Firstly, it has a relatively simple once-a-day, three-day dosage regimen and a bioavailability that does not require fat-rich meals [37]. This is likely to increase adherence to treatment, which will minimize wastage and improve therapeutic outcomes. Secondly, DhP has a long elimination half-life, which may give it a prolonged post-treatment prophylactic effect that would help to reduce future costs from recurrent infections [18].

In the base-case analysis, DhP was a dominant strategy based on the assumption that it has a compliance rate higher than that of AL; unfortunately, this has not been documented in clinical practice. Since the two drugs have similar safety profiles [13], and taking into account the complex dosage regimen and the pill burden of $\mathrm{AL}$, it is unlikely that the compliance rate for DhP will be lower than that of AL. In addition, the prolonged post-treatment prophylactic effect of DhP, which we did not consider in the analysis, would increase its costeffectiveness. A recent study has shown that DhP was strongly dominant over AL with a probability of $90 \%$, by modelling the differences in post-treatment prophylactic effect of the two drugs [18].

DhP has received regulatory approval from the European Medicines Agency (EMA) and can now be procured with donor funds [60], at an affordable maximum price of less than US\$ 1 per dose [29]. Sigma-Tau, the manufacturer of DhP (Eurartesim $\left.{ }^{\circ}\right)$, in collaboration with 


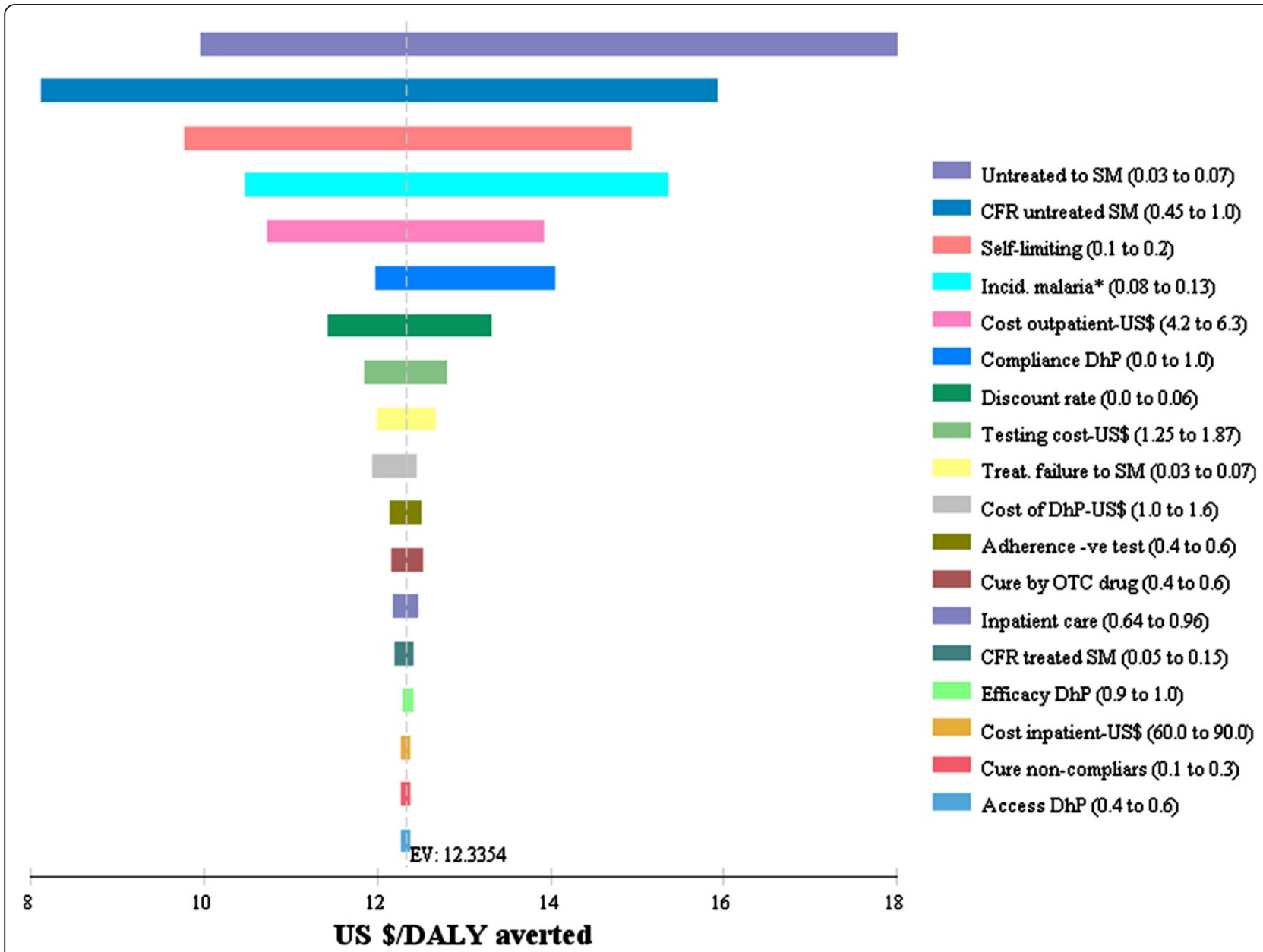

Figure 5 ICER tornado diagram of DhP compared to "do-nothing". Key: *Incidence rates as percentage of febrile episodes. Unless otherwise indicated, the numbers in the brackets represent probabilities

Medicine for Malaria Venture, are also developing a new water-dispersible formulation for children under the age of five years [61]. With generic competition, the price of DhP is likely to decrease even further over the coming years.

Even though DhP is a very promising long-acting anti-malarial drug, concerns have been raised about its residual drug levels as a potential risk for the emergence of resistance, especially in high transmission areas $[62,63]$. A reliable surveillance system is therefore needed to monitor its therapeutic efficacy [13]. Several studies have also shown that the administered dosage and the resulting plasma concentrations are the most important predictors of treatment failures in children treated with DhP $[64,65]$. Thus, malaria experts have suggested increasing the minimum dosage of piperaquine recommended by the WHO from 48 to $59 \mathrm{mg} / \mathrm{kg}$ in order to achieve desirable plasma concentrations [64].

Presumptive treatments and non-adherence to negative test results is another common challenge facing the deployment of expensive drugs like DhP in endemic countries. Studies in Tanzania have shown that malaria is highly over diagnosed and non-adherence to negative test results may be as high as 53\% [56]. The WHO's malaria report of 2011 showed that perfect compliance with negative test results would save US\$ 68 million by eliminating the unnecessary use of ACT in the public sector in Africa [66].

\section{Limitations}

The study used the two-week self-reported prevalence of fever from a national survey to estimate the weekly incidence rates of febrile episodes in children [50]. This approach can overestimate or underestimate the actual incidence rates given the seasonal variation of fever episodes and when counting is not precise due to the overlapping of fevers during the two-week period. This is, however, a preferred approach in the absence of systematically collected data about the annual incidence rates of febrile episodes [66]. 


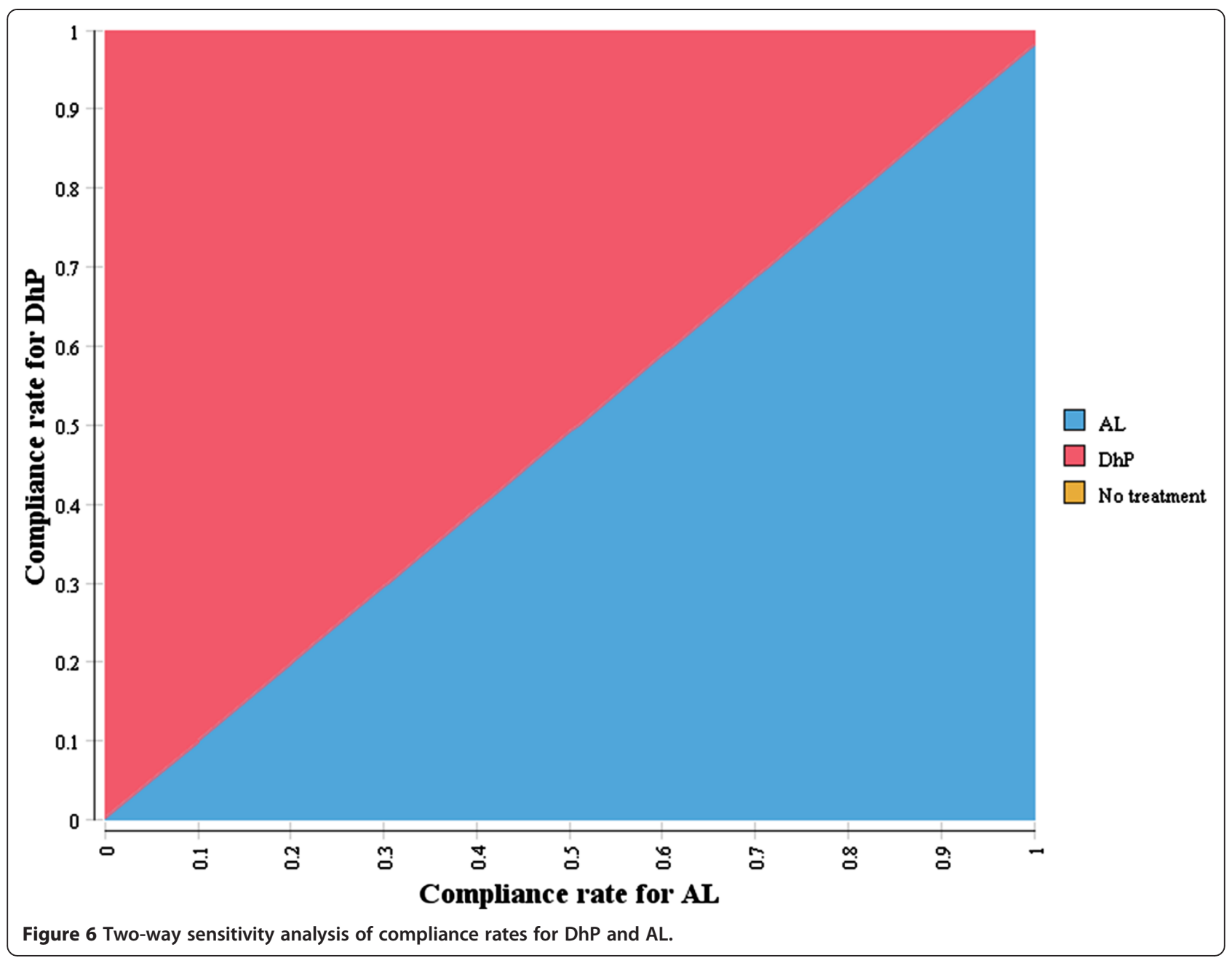

Pragmatic costing studies are difficult to undertake in low-income countries because resource use and attendances at specific departments are not always properly documented. Therefore, we did not include costs for consumables, such as cannulas, syringes, cotton wool and infusion sets. It was also very challenging to allocate overhead costs to service departments, and this forced us to use weighted factors. It was also difficult to adequately calculate unit costs for laboratory and pharmacy services because attendances at these units were not properly recorded. Therefore, the estimated unit costs may have underestimated the actual treatment costs for uncomplicated and severe malaria.

The study was conducted from a provider's perspective without including a more comprehensive societal perspective. Unlike DhP, the use of AL is associated with greater costs due to the requirement for fat-rich meals to optimize its bioavailability. A societal perspective may, therefore, increase treatment costs relatively more for $\mathrm{AL}$, thus favouring $\mathrm{DhP}$ in the costeffectiveness analysis.
The study was focused on health losses due to malaria only, therefore, DALYs lost from the associated comorbidities of severe malaria such as anemia, convulsions and long-term neurological injury were not included in the model. Their inclusion would have favoured DhP in the cost-effectiveness analysis, because it is relatively more effective than AL due to its high efficacy and compliance rates.

\section{Generalizability}

The study used AL, which is the current first-line antimalarial drug against uncomplicated malaria in many malaria-endemic countries, as a comparator. The drug prices also include freight and insurance charges as well as local administrative costs, which to a large extent accommodate uncertainties in supplier prices. Given that the results were robust to plausible variations in all the key parameters, they are likely to be relatively generalizable to other settings with similar healthcare infrastructures and malaria epidemiology. 


\section{Conclusion}

DhP is a very cost-effective anti-malarial drug. The findings support its use as an alternative first-line drug for treating uncomplicated malaria in children in Tanzania and other sub-Saharan African countries with similar healthcare infrastructures and malaria epidemiology. A number of countries in malaria-endemic areas are currently considering the adoption of DhP in their malaria treatment guidelines. Therefore, policy-makers in these countries should employ this evidence in order to make informed decisions about allocating their limited resources to competing healthcare interventions.

\section{Additional file}

Additional file 1: Personnel costs and rental charges.

\section{Abbreviations}

ACT: Artemisinin-based combination therapies; AL: Artemether-lumefantrine AMFm: Affordability medicines facility-malaria; DALY: Disability adjusted life years; DhP: Dihydroartemisinin-piperaquine; MSD: Medical stores department.

\section{Competing interests}

The authors declare that they have no competing interests.

\section{Authors' contributions}

ATM, FN, OFN and BR conceived the study. ATM collected the data and performed the analysis. ATM and BR prepared and designed the economic model. ATM and FN prepared and revised the first draft of the manuscript. $\mathrm{BR}$ and OFN supervised data collection and analysis and contributed to the manuscript writing. All authors read and approved the final manuscript.

\section{Acknowledgements}

We thank the Priority-Setting Research Group for their constructive comments on early drafts of the manuscript. We also thank the staff at Mwananyamala Hospital for the support they gave us during the collection of cost data. We also thank the Norwegian State Education Loan Fund (Statens Lånekasse) and the University of Bergen, which provided funding for this study. The funders had no role in the study design, data collection and analysis, decision to publish, or preparation of the manuscript.

\section{Author details}

${ }^{1}$ Centre for International Health, Department of Global Public Health and Primary Care, University of Bergen, P.O. Box 7804, 5020 Bergen, Norway. ${ }^{2}$ Muhimbili University of Health and Allied Sciences, P.O. Box 65001, Dar es Salaam, Tanzania. ${ }^{3}$ Ministry of Health and Social Welfare, P.O. Box 9083, Dar es Salaam, Tanzania.

Received: 7 June 2014 Accepted: 12 September 2014 Published: 15 September 2014

\section{References}

1. WHO: World Malaria Report. Geneva: World Health Organization; 2013.

2. Murray CJ, Global Burden of Disease Study Group: Disability-adjusted life years (DALYs) for 291 diseases and injuries in 21 regions, 1990-2010: a systematic analysis for the Global Burden of Disease Study 2010. Lancet 2012, 380:2197-2223.

3. UNICEF, RBM: Malaria and Children: Progress in intervention coverage. New York: United Nations Children's Fund and Roll Back Malaria; 2007.

4. WHO: Guidelines for the Treatment of Malaria. 2nd edition. Geneva: World Health Organization; 2010.

5. Sinclair D, Zani B, Donegan S, Olliaro P, Garner P: Artemisinin-based combination therapy for treating uncomplicated malaria. Cochrane Database Syst Rev 2009, 3:CD007483.

6. Naing C, Mak JW, Aung K, Wong J: Efficacy and safety of dihydroartemisinin-piperaquine for treatment of uncomplicated
Plasmodium falciparum malaria in endemic countries: meta-analysis of randomized controlled trials. Trans R Soc Trop Med Hyg 2012, 107:65-73. 7. Kamya MR, Yeka A, Bukirwa H, Lugemwa M, Rwakimari JB, Staedke SG, Talisuna AO, Greenhouse B, Nosten F, Rosenthal PJ, Wabwire-Mangen F, Dorsey G: Artemether-lumefantrine versus dihydroartemisinin-piperaquine for treatment of malaria: a randomized trial. PLOS Clin Trials 2007, 2:e20.

8. Yeka A, Dorsey G, Kamya MR, Talisuna A, Lugemwa M, Rwakimari JB, Staedke SG, Rosenthal PJ, Wabwire-Mangen F, Bukirwa H: Artemetherlumefantrine versus dihydroartemisinin-piperaquine for treating uncomplicated malaria: a randomized trial to guide policy in Uganda. PLoS One 2008, 3:e2390.

9. Nambozi M, Geertruyden JP, Hachizovu S, Chaponda M, Mukwamataba D, Mulenga M, Ubben D, D'Alessandro U: Safety and efficacy of dihydroartemisinin-piperaquine versus artemether-lumefantrine in the treatment of uncomplicated plasmodium falciparum malaria in Zambian children. Malar J 2011, 10:50.

10. Karema C, Fanello Cl, Overmeir CV, JP V g, van Doren W, Ngamije D, D'Alessandro U: Safety and efficacy of dihydroartemisinin/piperaquine (Artekin $^{\oplus}$ ) for the treatment of uncomplicated plasmodium falciparum malaria in Rwandan children. Trans R Soc Trop Med Hyg 2006, 100:1105-1111.

11. Bassat $Q$, Mulenga M, Tinto H, Piola P, Borrmann S, Mene'ndez C, Nambozi M, Vale'a I, Nabasumba C, Sasi P, Bacchieri A, Corsi M, Ubben D, Talisuna A, D'Alessandro U: Dihydroartemisinin-piperaquine and artemetherlumefantrine for treating uncomplicated malaria in african children: a randomised, non-inferiority trial. PLOS One 2009, 4:e7871.

12. Adam I, Salah MT, Eltahir HG, Elhassan AH, Elmardi KA, Malik EM: Dihydroartemisinin-piperaquine versus artemether-lumefantrine, in the treatment of uncomplicated plasmodium falciparum malaria in central Sudan. Ann Trop Med Parasitol 2010, 104:319-326.

13. The Four Artemisinin-Based Combinations (4ABC) Study Group: A head-to-head comparison of four artemisinin-based combinations for treating uncomplicated malaria in african children: a randomized trial. PLoS Med 2011, 8:e1001119.

14. Ministry of Health and Social Welfare: National Guidelines for Malaria Diagnosis and Treatment. Dar es Salaam: National Malaria Control Programme; 2006.

15. Ministry of Health and Social Welfare: Standard Treatment Guidelines and National Essential Medicine List-Tanzania Mainland. 4th edition. Dar es Salaam: Ministry of Health and Social Welfare; 2013.

16. Wiseman V, Kim M, Mutabingwa TK, Whitty CJ: Cost-effectiveness study of three antimalarial drug combinations in Tanzania. PLoS Med 2006, 3:e373.

17. Chanda P, Masiye F, Chitah BM, Sipilanyambe N, Hawela M, Banda P, Okorosobo T: A cost-effectiveness analysis of artemether lumefantrine for treatment of uncomplicated malaria in Zambia. Malar J 2007, 6:21.

18. Pfeil J, Borrmann S, Tozan Y: Dihydroartemisinin-piperaquine vs. artemether-lumefantrine for first-line treatment of uncomplicated malaria in african children: a cost-effectiveness analysis. PLoS One 2014, 9:e95681.

19. Ministry of Health: Guidelines for Case Management of Malaria in Ghana. 2nd edition. Accra: Ministry of Health; 2009.

20. Ministry of Public Health and Sanitation: National Guidelines for the Diagnosis, Treatment and Prevention of Malaria in Kenya. 3rd edition. Nairobi: Ministry of Public Health and Sanitation; 2010.

21. Mori AT, Gavaza P, Robberstad B: The role of pharmacoeconomics in developing countries. Farmeconomia. Health Economics and Therapeutic Pathways 2013, 14:3-5.

22. Gatton ML, Cheng Q: Modeling the development of acquired clinical immunity to plasmodium falciparum malaria. Infect Immun 2004, 72:6538-6545

23. Miller LH, Good MF, Milon G: Malaria pathogenesis. Science 1994, 264:1878-1884.

24. Drummond MF, Sculpher MJ, Torrance GW, O'Brien BJ, Stoddart GL: Methods for the Economic Evaluation of Health Care Programmes. 3rd edition. New York: Oxford University Press; 2005.

25. Bank of Tanzania: Economic Bulletin for the Quarter Ending December 2012. Economic Bulletin; 2012:XLIV.

26. WHO: Cost Analysis in Primary Health Care: A training Manual for Programme Managers. Geneva: World Health Organization; 1994.

27. Medical Stores Department: Price Catalogue of Essential Medicines, Diagnostics and Hospital Supplies-2011/2012. Dar es Salaam: Medical Stores Department. 
28. WHO: Making Choices in Health: WHO Guide to Cost-Effectiveness Analysis. Geneva: World Health Organization; 2003.

29. The Global Fund: ACT Prices under the Affordable Medicines Facility-malaria: Update of 22 May 2013. Geneva, Switzerland: The Global Fund; 2013.

30. Sigma-Tau SA: Application for Inclusion of Dihydroartemisinin/Piperaquine in the 17th WHO Model List of Essential Medicines; 2010.

31. Management Science for Health: International Drug Price Indicator Guide. 2011th edition. Arlington, USA: Management Science for Health, Inc; 2012.

32. Johns B, Baltussen R, Hutubessy R: Programme costs in the economic evaluation of health interventions. Cost Effect Resource Alloc 2003, 1:1.

33. Salomon JA, Global Burden of Disease Group: Common values in assessing health outcomes from diseases and injury: disability weights measurement study for the Global Burden of Disease Study 2010. Lancet 2012, 380:2129-2143.

34. Fox-Rushby JA, Hanson K: Calculating and presenting disability adjusted life years (DALYs) in cost-effectiveness analysis. Health Policy Plan 2001 16:326-331

35. WHO: World Health Statistics 2012: Life Tables for WHO Member States. Geneva: World Health Organization; 2012.

36. Ezzet F, Vugt M, Nosten F, Looareesuwan S, White NJ: Pharmacokinetics and pharmacodynamics of lumefantrine (Benflumetol) in acute falciparum malaria. Antimicrob Agents Chemother 2000, 44:697-704.

37. Tarning J, Lindegardh N, Lwin KM, Annerberg A, Kiricharoen L, Ashley E, White NJ, Nosten F, Day NPJ: Population pharmacokinetic assessment of the effect of food on piperaquine bioavailability in patients with uncomplicated malaria. Antimicrob Agents Chemother 2014, 58:2052-2058.

38. Zani B, Gathu M, Donegan S, Olliaro PL, Sinclair D: Dihydroartemisininpiperaquine for treating uncomplicated Plasmodium falciparum malaria. Cochrane Database Syst Rev 2014, 1:CD010927.

39. Coleman P, Morel C, Shillcut S, Goodman C, Mills A: A threshold analysis of the cost-effectiveness of artemisinin-based combination therapies in sub-Saharan Africa. Am J Trop Med Hyg 2004, 7:196-204.

40. Committee on the Economics of Antimalarial Drugs, Board on Global Health: The cost and cost-effectiveness of antimalarial drugs. In Saving Lives, Buying Time: Economics of Malaria Drugs in an Age of Resistance. Edited by Arrow KJ, Panosian CB, Gelband H. Washngton, DC: National Academies Press; 2004:61-78.

41. Goodman C, Coleman P, Mills A: Economic Analysis of Malaria Control in Sub-Saharan Africa. Geneva: Global Forum for Health Research; 2000.

42. Banek K, Lalani M, Staedke SG, Chandramohan D: Adherence to artemisinin-based combination therapy for the treatment of malaria: a systematic review of the evidence. Malar J 2014, 13:7.

43. Bruxvoort K, Goodman C, Kachur SP, Schellenberg D: How patients take malaria treatment: a systematic review of the literature on adherence to antimalarial drugs. PLoS One 2014, 9:e84555

44. Beer N, Ali AS, Rotllant G, Abass AK, Omari RS, Al-mafazy AH, Bjo "rkman A, Ka"llander K: Adherence to artesunate-amodiaquine combination therapy for uncomplicated malaria in children in Zanzibar, Tanzania. Trop Med Inter Health 2009, 14:766-774.

45. Ratsimbasoa A, Ravony H, Vonimpaisomihanta JA, Raherinjafy $R$, Jahevitra M, Rapelanoro R, Rakotomanga JM, Malvy D, Millet P, Ménard D: Compliance, safety, and effectiveness of fixed-dose artesunate-amodiaquine for presumptive treatment of non-severe malaria in the context of home management of malaria in Madagascar. Am J Trop Med Hyg 2012, 86:203-210.

46. D'Acremont V, Kilowoko M, Kyungu E, Philipina S, Sangu W, Kahama J, Lengeler C, Cherpillod P, Kaiser L, Genton B: Beyond malaria - causes of fever in outpatient Tanzanian children. N Engl J Med 2014, 370:809-817.

47. Mugittu K, Abdulla S, Falk N, Masanja H, Felger I, Mshinda H, Beck H, Genton B: Efficacy of sulfadoxine-pyrimethamine in Tanzania after two years as first-line drug for uncomplicated malaria: assessment protocol and implication for treatment policy strategies. Malar J 2005, 4:55.

48. Mutabingwa TK, Anthony D, Heller A, Hallett R, Ahmed J, Drakeley C, Greenwood BM, Whitty CJ: Amodiaquine alone, amodiaquine + sulfadoxine-pyrimethamine, amodiaquine + artesunate, and artemetherlumefantrine for outpatient treatment of malaria in Tanzanian children: a four-arm randomised effectiveness trial. Lancet 2005, 365:1474-1480.

49. WHO: World Health Statistics. Geneva: World Health Organization; 2012

50. Tanzania Commission for AIDS, Zanzibar AIDS Commission, National Bureau of Statistics, The Chief Government Statistician, ICF International:: HIV/AIDS and Malaria Indicator Survey 2011-2012. Dar es Salaam: TACAIDS, ZAC, NBS, OCGS and ICF International; 2013.

51. Lubell Y, Staedke SG, Greenwood BM, Kamya MR, Molyneux M, Newton PN, Reyburn H, Snow RW, D'Alessandro U, English M, Day N, Kremsner P, Dondorp A, Mbacham W, Dorsey G, Owusu-Agyei S, Maitland K, Krishna S, Newton C, Pasvol G, Taylor T, von Seidlein L, White NJ, Binka F, Mills A, Whitty CJ: Likely health outcomes for untreated acute febrile illness in the tropics in decision and economic models: a Delphi survey. PLoS One 2011, 6:e17439.

52. Dondorp AM, Fanello Cl, Hendriksen IC, Gomes E, Seni A, Chhaganlal KD, Bojang K, Olaosebikan R, Anunobi N, Maitland K, Kivaya E, Agbenyega T, Nguah SB, Evans J, Gesase S, Kahabuka C, Mtove G, Nadjm B, Deen J, Amumpaire JM, Nansumba M, Karema C, Umulisa N, Uwimana A, Mokuolu OA, Adedoyin OT, Johnson WB, Tshefu AK, Onyamboko MA, Sakulthaew T, Ngum WP, Silamut K, Stepniewska K, Woodrow CJ, Bethell D, Wills B, Oneko M, Peto TE, von Seidlein L, Day NP, White NJ, for the AQUAMAT group: Artesunate versus quinine in the treatment of severe falciparum malaria in African children (AQUAMAT): an open-label, randomised trial. Lancet 2010, 13:1647-1657.

53. De Savigny D, Mayombana C, Mwageni E, Masanja H, Minhaj A, Mkilindi Y, Mbuya C, Kasale H, Reid G: Care-seeking patterns for fatal malaria in Tanzania. Malar J 2004, 3:27.

54. Tan-Torres E, Baltussen R, Adam T RH, Acharya A, Evans DB, Murray CJ: Making choices in health care: WHO guide to cost-effectiveness analysis. Geneva: World Health Organization; 2003.

55. Shillcutt SD, Walker DG, Goodman CA, Mills AJ: Cost-effectiveness in low- and middle-income countries: a review of the debates surrounding decision rules. Pharmacoeconomics 2009, 27:903-917.

56. Reyburn H, Mbakilwa H, Mwangi R, Mwerinde O, Olomi R, Chris Drakeley C, Whitty CJM: Rapid diagnostic tests compared with malaria microscopy for guiding outpatient treatment of febrile illness in Tanzania: randomised trial. BMJ 2007, 334:403.

57. Briggs A, Claxton K, Sculpher M: Decision Modelling for Health Economic Evaluation. Oxford: Oxford University Press; 2006

58. Cantor SB: Cost-effectiveness analysis, extended dominance, and ethics: a quantitative assessment. Med Decis Making 1994, 14:259-265.

59. WHO: World Health Report. Geneva, Switzerland: World Health Organization; 2002.

60. Ubben D, Poll EM: MMV in partnership: the Eurartesim experience. Malar J 2013, 12:211.

61. Medicines for Malaria Venture: Eurartesim $*$ : Stamp of approval from the European Medicines Agency. 2011. Available on: http://www.mmv.org/ achievements-challenges/achievements/eurartesim ${ }^{\oplus}$.

62. Arinaitwe E, Sandison TG, Wanzira H, Kakuru A, Homsy J, Kalamya J, Kamya MR, Vora N, Greenhouse B, Rosenthal PJ, Tappero J, Dorsey G: Artemether-lumefantrine versus dihydroartemisinin-piperaquine for falciparum malaria: a longitudinal, randomized trial in young Ugandan children. Clin Infect Dis 2009, 49:1629-1637.

63. White NJ: How antimalarial drug resistance affects post-treatment prophylaxis. Malar J 2008, 7:9.

64. The WorldWide Antimalarial Resistance Network (WWARN) DP Study Group: The effect of dosing regimens on the antimalarial efficacy of dihydroartemisinin-piperaquine: a pooled analysis of individual patient data. PLoS Med 2013, 10:e1001564.

65. Price RN, Hasugian AR, Ratcliff A, Siswantoro H, Purba HL, Kenangalem E, Lindegardh N, Penttinen P, Laihad F, Ebsworth EP, Anstey NM, Tjitra E: Clinical and pharmacological determinants of the therapeutic response to dihydroartemisinin-piperaquine for drug-resistant malaria. Antimicrob Agents Chemother 2007, 51:4090-4097.

66. WHO: World Malaria Report. Geneva: World Health Organization; 2011.

67. Gething PW, Kirui VC, Alegana VA, Okiro EA, Noor AM, Snow RW: Estimating the number of paediatric fevers associated with malaria infection presenting to Africa's public health sector in 2007. PLoS Med 2010, 7:e1000301.

doi:10.1186/1475-2875-13-363

Cite this article as: Mori et al: Cost-effectiveness of

dihydroartemisinin-piperaquine compared with artemether-

lumefantrine for treating uncomplicated malaria in children at a district hospital in Tanzania. Malaria Journal 2014 13:363. 